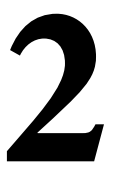

\title{
MODELO DE ANÁLISIS DE METODOLOGÍAS DIDÁCTICAS SEMIPRESENCIALES EN EDUCACIÓN SUPERIOR
}

\author{
(MODEL FOR ANALYZING BLENDED LEARNING IN HIGHER EDUCATION)
}

Vanesa Gámiz Sánchez

María Jesús Gallego Arrufat

Universidad de Granada

DOI: $10.5944 / e d u c X X 1.15577$

Cómo referenciar este artículo/How to reference this article:

Gámiz- Sánchez, V. y Gallego-Arrufat, M.J. (2016). Modelo de análisis de metodologías didácticas semipresenciales en Educación Superior. Educación XX1, 19(1), 39-61, doi:10.5944/ educXX1.15577

Gámiz- Sánchez, V. \& Gallego-Arrufat, M.J. (2016). Modelo de análisis de metodologías didácticas semipresenciales en Educación Superior [Model for analyzing blended learning in Higher Education]. Educación XX1, 19(1), 39-61, doi:10.5944/educXX1.15577

\section{RESUMEN}

En esta investigación experimentamos un modelo multidimensional para el análisis de metodologías blended learning en Educación Superior. Incluye la opinión de estudiantes de Educación sobre la formación online y el papel del profesorado, y un modelo basado en las categorías: actuación del estudiante; actuación del docente; opinión sobre la modalidad de aprendizaje; y estadísticas de acceso y uso de la plataforma. Comprobamos que las metodologías didácticas basadas en el blended learning pueden resultar beneficiosas para los cambios que se están realizando en la Educación Superior en su proceso de convergencia hacia el EEES, según la opinión de los estudiantes antes y después de desarrollar un proceso de aprendizaje semipresencial, además de cuantificar la relación entre la participación, según las estadísticas de acceso, y sus resultados académicos. A través de un enfoque mixto obtenemos datos cuantitativos (items escala likert en cuestionarios) y cualitativos (foros y preguntas abiertas) que nos llevan a comprobar que, según la perspectiva de los estudiantes, es un camino más favorable que el tradicional, si bien cabe reflexionar sobre los aspectos negativos (entre los que destaca que los estudiantes consideran que el trabajo realizado en una asignatura semipresencial 
es significativamente mayor), debiendo adecuar principios metodológicos y organizativos en la Educación Superior a estas nuevas realidades.

\section{PALABRAS CLAVE}

Formación; universidad; métodos de enseñanza; enseñanza semipresencial; tecnología educativa; uso didáctico del ordenador.

\section{ABSTRACT}

In this research we test a multidimensional model for analyzing blended learning methodologies in Higher Education. This includes the opinion of Education students about online learning and the role of teachers in the same. It also includes their opinion about a model based on the following categories: student performance; teacher performance; opinion about the method of learning; and some statistics of platform access and use. We verify that blended learning methodologies can be useful in the changes carried out in the Higher Education institutions in their way towards the European Higher Education Area (EHEA). We can check this statement by studying students opinions before and after the development of a blended learning process, by relating the participation of the students and their learning outcomes. Through a mixed approach we collect quantitative data (Likert scale items in questionnaires) and qualitative data (forum and open questions). These data confirm that according to the students, this path of learning is more positive than the traditional one, though we have to reflect on negatives issues. Between these unfavorable issues, students highlight that the workload in a blended learning experience is considerably higher than in a traditional face to face process. Because of this we must think about adjusting methodological and organizational principles in Higher Education to these new realities.

\section{KEY WORDS}

Training; higher education; teaching methods; blended learning; educational technology; computer uses in education.

\section{INTRODUCCIÓN}

En este artículo se analiza la opinión de los estudiantes sobre un modelo de aprendizaje combinado o blended learning en varias asignaturas en la Facultad de Ciencias de la Educación de la Universidad de Granada. Se pretende conocer sus actitudes de partida ante la docencia semipresencial y las opiniones que tienen después de experimentar metodologías que combi- 
nan enseñanza presencial y online. Estas metodologías están cada vez más extendidas tanto a nivel nacional como europeo y en educación formal y no formal (Graham, 2006).

A nivel europeo, la Estrategia Europa 2020 invita a los estados europeos a colaborar con los centros de educación superior para promover la innovación a través de entornos de aprendizaje más interactivos. Las conclusiones del Consejo de Europa sobre la modernización de la enseñanza superior incluyen explícitamente fomentar la adopción de enfoques centrados en el estudiante, promoviendo la diversificación de las modalidades de estudio, y también haciendo un uso eficaz de las tecnologías de la información y de las comunicaciones (Diario Oficial de la Unión Europea, 20/12/2011).

A nivel nacional, en el último Informe de la CRUE, aumentan las cifras en los objetivos «proporcionar soporte e introducir nuevas tecnologías de apoyo a la docencia presencial» y «proporcionar soporte y promover la docencia no presencial». Un número medio de 1.502 PDI por universidad emplean la plataforma de docencia virtual institucional (87\% del total de docentes de cada institución). Por término medio, 21.730 estudiantes emplean la plataforma de docencia virtual de la institución (90,6\% de los estudiantes) (Uceda y Píriz, 2012, 21-22). Estos datos llevan a plantear si las metodologías combinadas pueden convertirse en un factor de cambio y de mejora de la universidad y para ello es preciso investigar la opinión del alumnado en este proceso y el alcance de su integración en el mismo. El alumnado como protagonista de su aprendizaje valora la metodología para comprobar si se corresponde en la práctica con lo que teóricamente se postula. El siguiente paso es averiguar en qué medida se pueden relacionar estas metodologías con los resultados de aprendizaje.

Encontramos en el contexto europeo interesantes modelos de análisis descriptivos. Fabian, Veen y Mühren (2008) aportan un modelo de investigación situada en el contexto con grupos numerosos de estudiantes $(\mathrm{N}>100)$, al que denominan BLEND-XL (http://www.blend-xl.eu/); Ryberg, Niemczik y Brenstein (2009) proyectan una wiki denominada Methopedia en el marco del European Community of Integrative Blended Learning Experts (COMBLE) (http://www.comble-project.eu), para compartir metodologías y actividades. Sin embargo, los estudios en los que se relacionan con el logro de los estudiantes no son concluyentes. En Turquía, Delialioglu y Yildirim (2008) realizaron un estudio experimental pre-test y post-test con 50 estudiantes universitarios ( 24 y 26 respectivamente) matriculados en una materia sobre Comunicación y redes de ordenadores durante 14 semanas. Trataron de comprobar la efectividad de la instrucción híbrida en cuanto a rendimiento de los estudiantes, retención de conocimiento, actitudes hacia el tema y 
satisfacción hacia el curso. Luego se evaluó en comparación con la instrucción tradicional en el aula. Los resultados del estudio no encontraron diferencias significativas entre el curso híbrido y el curso tradicional ni en el rendimiento de los estudiantes ni en el resto de variables.

En nuestro contexto, una investigación similar muestra que las percepciones de los estudiantes sobre el aprendizaje combinado se relacionan entre sí y con sus calificaciones finales. El efecto conjunto es explicativo de la reducción de las tasas de deserción escolar y el aumento de la nota final. La percepción de la utilidad de las actividades de e-learning por sí sola no afecta a la calificación final, por lo que se puede concluir que las actividades de e-learning, en lugar de reemplazar, deben complementar las formas tradicionales de aprendizaje (López, Pérez y Rodríguez, 2011; Mitchell y Forer, 2010). Ya anteriormente Ginns y Ellis (2009) habían apuntado a la importancia de comprender las percepciones de los estudiantes del ambiente de aprendizaje (más que la calidad técnica y funcional de los materiales online), y sugirieron que si el profesorado quiere que los estudiantes mejoren sus percepciones y sus calificaciones, obteniendo un rendimiento más elevado en el aprendizaje en línea en contextos mezclados, entonces las estrategias de enseñanza deberán dirigirse hacia la moderación y potenciar el valor de la interacción en las intervenciones online también en otros contextos, no solo en las plataformas de enseñanza virtual (Gallego y Gámiz, 2014). Si tiene lugar alguna forma de aprendizaje interactivo entonces será útil el incremento del tiempo destinado al estudio online (Castaño-Muñoz, Duart y Sancho-Vinuesa, 2013). También el nivel de compromiso del estudiante con la metodología de aprendizaje combinada mejora sus resultados (Francis y Shannon, 2013).

\section{METODOLOGÍA}

El estudio que se describe en este artículo forma parte de una investigación mayor, financiada por el Ministerio de Ciencia y Tecnología en el Plan Nacional de I+D+i (España), Ref. SEJ2004-08062-C02-00, dirigido a la experimentación con una plataforma de enseñanza virtual en un modelo blended learning con estudiantes de Ciencias de la Educación.

El diseño de la investigación consta de tres fases: inicial, en la que se sondea la opinión de los estudiantes antes del uso de metodologías blended learning, con un método descriptivo-exploratorio; la fase de desarrollo, con un enfoque cuasiexperimental; y una fase final, en la que se pregunta a los 
estudiantes sobre el desarrollo de la experiencia y sus resultados a través de un método descriptivo-explicativo.

El enfoque metodológico es mixto o multimétodo, tanto en la recogida como en el análisis de datos, predominando una perspectiva cuantitativa en la primera y la última fase y extrayendo los datos a través de cuestionarios diseñados a tal efecto, mientras que en la fase de desarrollo predomina la perspectiva cualitativa, analizando foros, chats y opiniones de los estudiantes. Con ello realizamos una triangulación en los métodos de recogida de datos y en las fuentes emisoras, persiguiendo con ello el aumento en la validez (Campbell y Fiske, 1959; Webb, Campbell, Schwartz y Sechrest, 1966), entendida como el grado de adecuación de una medida particular respecto a la realidad que apunta.

En esta investigación se obtiene información de varias fuentes que constituyen poblaciones diferenciadas y que aportan información complementaria. De un lado, estudiantes realizando su periodo de prácticum siguiendo la supervisión blended learning y estudiantes que siguen un modelo de supervisión tradicional y, de otro lado, estudiantes de otras asignaturas regladas que emplean la plataforma AulaWeb siguiendo la misma metodología con la misma herramienta formativa. La selección de los estudiantes de cada grupo se realiza través de un muestreo no probabilístico siguiendo criterios de conveniencia.

Como instrumentos de recogida de datos se utiliza, en la primera fase, el «Cuestionario Inicial para el Asesoramiento Virtual» (CIAV). Para la recopilación de información en la fase de desarrollo se usan los foros y las preguntas abiertas del «Cuestionario de Valoración de la Metodología didáctica con AulaWeb» (CVMA) sobre lo mejor y lo peor de la experiencia según los estudiantes. También se extraen estadísticas de participación de los estudiantes en la plataforma. En la tercera fase se utiliza el CVMA diseñado, al igual que el anterior, por el equipo de investigación del proyecto, validado por expertos.

La caracterización de la muestra y su relación con los instrumentos de recogida de datos y las fases de la investigación aparece en la Tabla 1. 
Tabla 1

Muestra, instrumentos y fases de la investigación

\begin{tabular}{|c|c|c|c|c|c|c|c|}
\hline & Asignatura & Especialidad & Curso & N. ${ }^{\circ}$ & Año & $\begin{array}{l}\text { Instru- } \\
\text { mento }\end{array}$ & Fase \\
\hline \multirow{4}{*}{$\begin{array}{l}\text { Estudiante } \\
\text { Prácticum } \\
\text { de Magis- } \\
\text { terio }\end{array}$} & \multirow{3}{*}{$\begin{array}{l}\text { Prácticum } \\
\text { Modali- } \\
\text { dad Semi- } \\
\text { presencial }\end{array}$} & \multirow{3}{*}{$\begin{array}{l}\text { Distintas } \\
\text { especialida- } \\
\text { des }\end{array}$} & \multirow[t]{3}{*}{$3 .^{\circ}$} & \multirow[t]{3}{*}{44} & \multirow[t]{3}{*}{ 2005-2009 } & CIAV & Inicial \\
\hline & & & & & & $\begin{array}{l}\text { Foros y } \\
\text { CVMA }\end{array}$ & Desarrollo \\
\hline & & & & & & CVMA & Final \\
\hline & $\begin{array}{l}\text { Prácticum } \\
\text { Modadli- } \\
\text { dad Tradi- } \\
\text { cional }\end{array}$ & $\begin{array}{l}\text { Distintas } \\
\text { especialida- } \\
\text { des }\end{array}$ & $3 .^{\circ}$ & $\begin{array}{c}26 \\
5\end{array}$ & 2007-2009 & $\begin{array}{l}\text { Foros y } \\
\text { CVMA }\end{array}$ & Desarrollo \\
\hline \multirow{3}{*}{$\begin{array}{l}\text { Estudiante } \\
\text { Ciencias } \\
\text { Educa- } \\
\text { ción }\end{array}$} & \multirow{3}{*}{$\begin{array}{l}\text { Tecnología } \\
\text { Educativa } \\
\text { y NNTT } \\
\text { aplicadas } \\
\text { a Educa- } \\
\text { ción }\end{array}$} & \multirow{3}{*}{$\begin{array}{l}\text { Pedagogía y } \\
\text { Magisterio }\end{array}$} & \multirow[t]{3}{*}{$2 .^{\circ} \mathrm{y} 3 .^{\circ}$} & \multirow{3}{*}{$\begin{array}{c}17 \\
5\end{array}$} & \multirow[t]{3}{*}{ 2007-2009 } & Foros y & Desarrollo \\
\hline & & & & & & CVMA & \\
\hline & & & & & & $\begin{array}{l}\text { CVMA y } \\
\text { Estadís- } \\
\text { tica }\end{array}$ & Final \\
\hline
\end{tabular}

El programa formativo llevado a cabo en la supervisión del prácticum descansa por un lado en la adquisición de competencias prácticas por parte de los estudiantes de Educación basadas en la práctica reflexiva, y, por otro, en el papel que puede tener una plataforma de e-learning como AulaWeb. Este programa consta de dos segmentos presenciales y dos virtuales dando lugar a un tipo de formación híbrida, mezclada o b-learning que describimos en detalle en otro lugar (Gallego y Gámiz, 2007).

En el modelo semipresencial empleado para asignaturas diferentes del prácticum se utiliza igualmente la plataforma en procesos de apoyo a la docencia presencial, haciendo especial hincapié en el trabajo colaborativo realizado de manera virtual a través de las Comunidades de prácticas, los ejercicios de autoevaluación para autorregular el aprendizaje de la parte teórica de las materias y la dinamización de las principales herramientas de comunicación grupales (foros y chat).

\section{RESULTADOS Y DISCUSIÓN}

\section{Análisis de la opinión del estudiante sobre las metodologías online y semipresenciales: Valoración inicial}

En primer lugar, se presentan las opiniones del alumnado con respecto a las metodologías no presenciales y semipresenciales, sondeadas a 
través del cuestionario inicial CIAV. Este cuestionario fue cumplimentado por todos los estudiantes del prácticum de la muestra $(\mathrm{N}=309)$.

Opinión sobre la formación online

Las expectativas iniciales ante la supervisión semipresencial son moderadamente buenas. Mayoritariamente el alumnado en una escala del 1 (muy deficiente) al 4 (excelente) se posiciona en el valor 3 y casi a partes iguales entre el 2 y 4.

\section{Expectativas iniciales supervisión no \\ presencial}

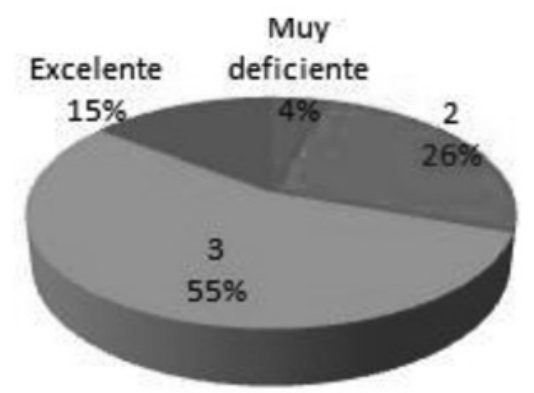

Figura 1. Expectativas de los estudiantes de prácticum ante una posible supervisión semipresencial

Rovai y otros (2006) detectan que la formación online levanta cierto recelo entre el alumnado hallando como resultado neto una calificación más negativa para los cursos en línea cuando comparan las valoraciones de los estudiantes de cursos presenciales en el campus y online impartidos por los mismos profesores.

Se pregunta también a los estudiantes sobre su opinión acerca del buen funcionamiento y desarrollo de un seminario/curso de formación con metodología semipresencial o no presencial. Bajo su perspectiva, esto dependerá, en primer lugar, de la predisposición y preparación del alumnado ( $98,8 \%$ en la suma de los valores «bastante» $\mathrm{y}$ «mucho»), seguido de la disponibilidad de recursos $(96,3 \%)$, y de la preparación del profesorado $(96,2 \%)$. En último lugar, el trabajo con otros compañeros $(79,2 \%)$ es lo menos importante para los encuestados como elemento determinante de éxito en la formación online. 


\section{Factores que influyen en la buena marcha de un} curso no presencial

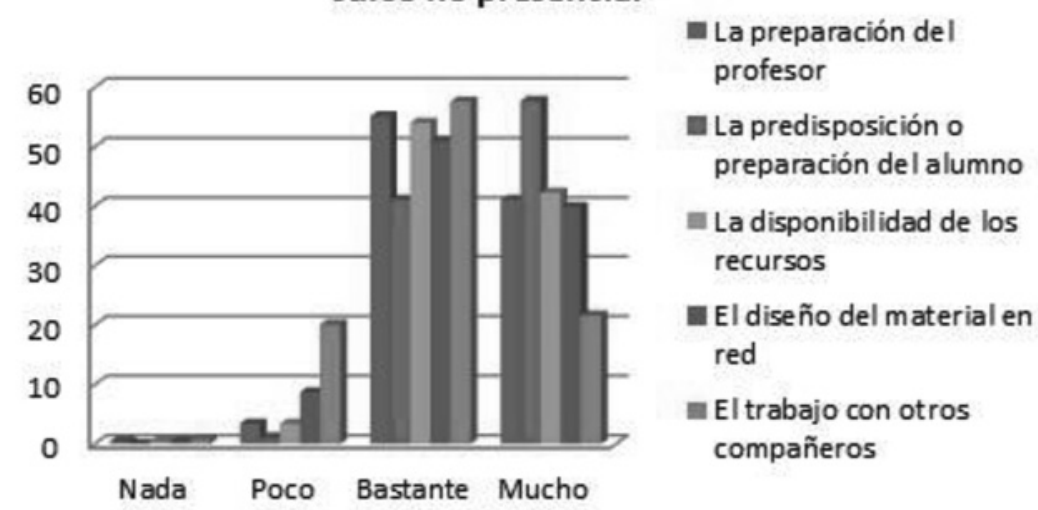

Figura 2. Factores que influyen en la buena marcha de un seminario o curso semipresencial o no presencial

En cuanto a la percepción sobre su preparación para afrontar un seminario/curso de estas características un $80,2 \%$ cree estar preparado frente a un $19,8 \%$ que cree no estarlo. Entre las razones aluden, en una escala del 1 (nada de acuerdo) al 4 (totalmente de acuerdo), que tienen los conocimientos informáticos iniciales básicos (55,6\% valor 3 y $27 \%$ valor 4$)$, se manejan bien aun no siendo especialistas en informática $(55,6 \%$ valor 3 y $27 \%$ valor 4$)$ y pueden aprender lo necesario (51,6\% valor 3 y $38,7 \%$ valor 4$)$. Se puede afirmar que 3 de cada 4 estudiantes se perciben a sí mismos como preparados para la enseñanza online, si bien con una formación autodidacta (Figura 3).

\section{Motivos por los que el estudiante cree estar preparado para la docencia online}

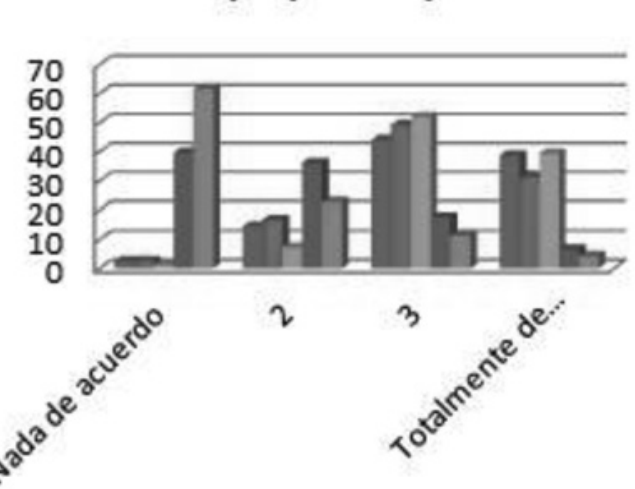
Tengo los conocim ientos básicos
Me manejo bien con la informática
Puedo aprender lo necesario

No tengo la información necesaria

Uso poco el ordenador

Figura 3. Razones por las que los estudiantes afirman mayoritariamente estar preparados para realizar un seminario/curso no presencial 
La predisposición del estudiante es un punto de partida clave. La renovación metodológica promovida por Bolonia incrementa la exigencia hacia los estudiantes y el profesorado (Lloret y Mir, 2007). Si para los estudiantes el aprendizaje activo requiere mayor dedicación e implicación, para el profesorado el nuevo enfoque metodológico demanda mayor preparación de la docencia y de los materiales y actividades asociadas a esta, mayor atención y seguimiento al proceso de aprendizaje de los estudiantes, y, en definitiva, supone nuevas necesidades técnicas y didácticas. Esta es también la opinión que manifiestan los estudiantes sobre las metodologías online.

\section{Papel del profesorado en la enseñanza online}

También se preguntó a los estudiantes si el profesorado cumple el mismo papel en la enseñanza presencial y en la no presencial o semipresencial. La opinión del alumnado sobre ello está bastante dividida y no parece decantarse claramente hacia la respuesta positiva $(52,3 \%)$ o negativa $(47,7 \%)$ (Figura 4).

\section{¿Es el papel del profesor el mismo que en la enseñanza presencial?}

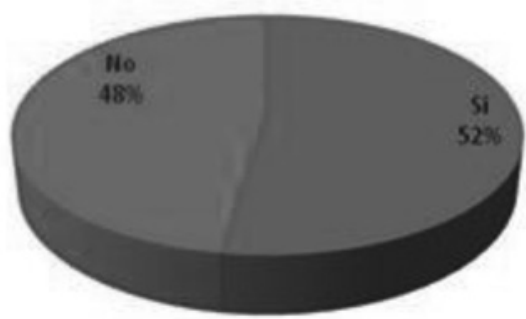

Figura 4. Comparación entre el papel del profesorado en la enseñanza presencial $y$ no presencial

En cuanto a los motivos de esta posición, valorando en una escala de 1 (nada de acuerdo) a 4 (totalmente de acuerdo), el 50,6\% del alumnado está totalmente de acuerdo en que el papel del profesor debería ser el mismo; el $49 \%$ considera que tanto en la enseñanza presencial como en la no presencial el profesor tiene las mismas funciones (orientación, transmisión de conocimientos, etc.); y el $42 \%$ considera que el profesor es igualmente intermediario de la información.

Las opciones menos valoradas son las referidas a la diferencia de roles en un modelo y otro. Tan sólo un 23,7\% opina que el docente debe ejercer 
más como mediador en la formación presencial; el 28,9\% considera que el papel es distinto, por el contacto directo cara a cara frente a la comunicación mediada por ordenador y solamente un 39,7\% están totalmente de acuerdo en que es distinto dirigir al grupo (presencial) que atender al estudiante individualmente (virtual).

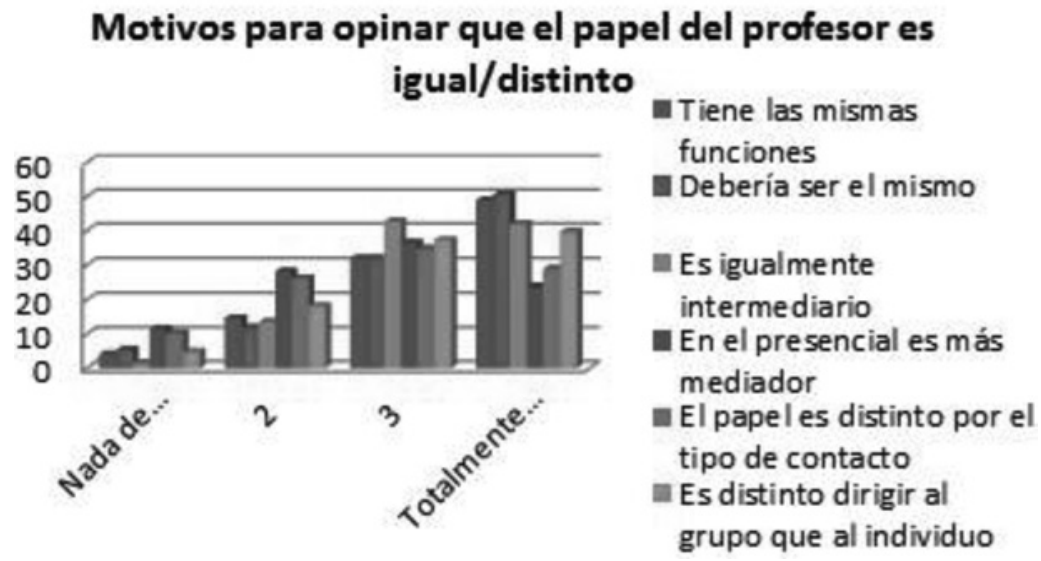

Figura 5. Motivos que alegan los estudiantes de prácticum para argumentar que el papel del docente es igual o distinto en las materias presenciales y no presenciales

Parece que los estudiantes perciben una falta de atención individualizada del profesor, característica sin duda muy importante de la enseñanza online. Sin embargo, en el sistema universitario actual teniendo en cuenta la masificación de las aulas es muy difícil poder conseguir esta individualización con éxito. Los estudiantes que entablan una comunicación y acción directa con el profesor a veces olvidan que en el otro sentido la interacción no es uno a uno, sino uno a muchos, lo que hace mucho más difícil la tarea de seguimiento. Cuando el grupo es más reducido el docente puede fomentar la participación y la interacción con mayor éxito (Lloret y Mir, 2007). Por otro lado, el cambio que debería existir en el rol del docente que trabaja en entornos tecnológicos, y que genera nuevas funciones como las de consultor, colaborador en grupo, facilitador del aprendizaje y generador crítico de conocimiento (Rovai et al., 2006; Álvarez, Guash y Espasa, 2009) no es percibido claramente por los estudiantes a priori.

\section{Análisis de la visión del estudiante sobre las metodologías online y semipresenciales: Valoración final}

A continuación se muestran datos sobre las opiniones de los estudiantes que realizaron créditos de prácticum o de materias de manera semipre- 
sencial, un total de 219 participantes. Se obtienen del cuestionario CVMA. Son sobre el aprendizaje que los estudiantes creen haber desarrollado, su percepción del trabajo del docente en la asignatura y otros aspectos relacionados con esta metodología.

\section{Actuación del estudiante}

Los estudiantes parecen tener patrones similares de respuesta en la comparación de la carga de trabajo en modelos presenciales y semipresenciales. El 33.3\% del alumnado encuestado otorga el máximo acuerdo a la afirmación de que su dedicación ha sido mayor. Este dato lleva a reflexionar sobre si la modalidad semipresencial, a pesar de flexibilizar los procesos de enseñanza-aprendizaje, puede ser considerada como una modalidad con más carga de trabajo y que, por lo tanto, requiere más tiempo y dedicación.

Otro ítem que obtiene valoración elevada puntúa la motivación e interés del estudiante en la asignatura (24,7\% valora con un 5 y $52,3 \%$ con un 4$)$. Con ello podemos pensar que a pesar de resultar una modalidad costosa en cuanto a esfuerzo, atrae su interés y consigue una predisposición bastante positiva. Por otra parte, el $74,7 \%$ de los encuestados cree que su aprendizaje combinado ha sido significativo $(59,8 \%$ lo valoran con un 4 y $14,9 \%$ con un 5) integrándose en su estructura cognitiva.

El estudiante percibe que el trabajo realizado en una asignatura semipresencial es mayor que el realizado en otras solo presenciales. Cuantificar la carga de la asignatura en las horas de trabajo del alumnado como exige el crédito europeo supone un reto para la organización y planificación docente y un mayor esfuerzo por parte de los estudiantes, de los cuales se exige una actitud más responsable y reflexiva. Las horas programadas por crédito ECTS según las directrices europeas quizá puedan ser excesivas para el estudiante en esta época de cambio (Montil y otros, 2007).

\section{Actuación del docente}

En cuanto a la opinión de los estudiantes sobre la actuación docente en la asignatura las respuestas también son bastante homogéneas y tienden a una media mayor de 4 (del 1 al 5). Muestran el mayor acuerdo sobre la coordinación estudiantes-profesorado-administrador de la plataforma, que califican mayoritariamente como buena $(51,2 \%)$ o muy buena $(20,3 \%)$, gracias a la efectividad de las herramientas de comunicación de la plataforma. Con bastante acuerdo, aunque en menor medida, perciben que la actuación docente va dirigida al desarrollo de competencias de búsqueda de información $(61,8 \%$ cree que Sí y 4,7\% opinan que Siempre) característica fundamental en estos modelos. También con un grado menor de acuerdo perciben 
que existe feedback en la interacción profesorado-estudiante (46,2\% cree que Si y 15,2\% opinan que Siempre). Quizá en este punto actúe una percepción distorsionada de las comunicaciones uno a muchos, ya que la inmediatez de una comunicación personal estudiante-profesor quiere verse correspondida con una comunicación instantánea profesor-estudiante, lo cual es sumamente difícil por el ratio profesor-estudiante y por la sobrecarga horaria de los profesores.

Es curiosa también la percepción que los estudiantes tienen del trabajo del profesor con respecto a la que tienen de su propio trabajo. A priori, podría creerse que más trabajo de los estudiantes conllevaría lógicamente más trabajo del profesor, pero no es entendido de esta manera por los estudiantes. Ellos tienen muy claro que su trabajo en la materia es mayor que en el resto de asignaturas pero no opinan claramente lo mismo como podemos observar en sus opiniones en la Figura 7. Existe una falta de valoración del trabajo docente no presencial al no ser tan «tangible» como el que se produce en horas de trabajo presencial.

\title{
Trabajo estudiante vs Trabajo profesor
}

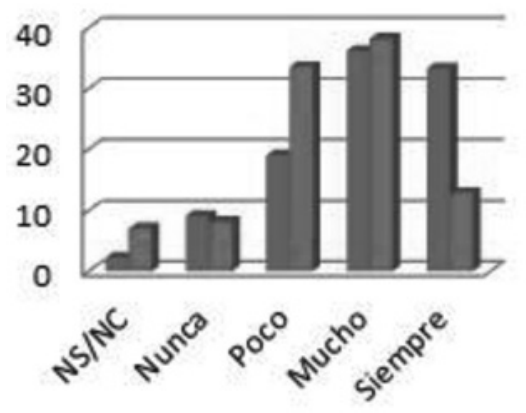

\author{
Estudiante trabaja \\ más \\ Profesortrabaja \\ más
}

Figura 7. Comparativa de la opinión del trabajo del estudiante con respecto al trabajo del profesor

Principalmente los estudiantes perciben la actuación del docente como mediador y guía en el proceso de aprendizaje, para lo cual la comunicación y la coordinación entre todas las partes debe ser determinante (Álvarez, Guasch y Espasa, 2009).

\section{Opinión sobre la modalidad de aprendizaje combinado}

Los estudiantes muestran un acuerdo elevado en prácticamente todos los ítems (media superior a 3,5). La dispersión de las respuestas tampoco es elevada mostrando todos los estudiantes una tendencia muy similar en sus respuestas. Las valoraciones más elevadas corresponden a la gran can- 
tidad de tiempo y esfuerzo empleados en desarrollar esta metodología (con un $28,7 \%$ y un $26,4 \%$ con valoración de 5 respectivamente) destacándolas como unas de las características más relevantes.

El acuerdo con las cuestiones propuestas también sigue siendo bastante elevado en la participación y trabajo práctico en la asignatura, otorgando un 77,8\% de los estudiantes valores de 4 y 5 al ítem que afirma que esta metodología facilita el desarrollo de habilidades prácticas a nivel general y un $70,7 \%$ (valores 4 y 5 ) al que señala que esta metodología favorece la participación activa. Consideran, pues, que con esta modalidad y a través de la plataforma es más sencillo que se participe en las distintas actividades propuestas y que sirva para desarrollar actividades prácticas.

Otros aspectos importantes son los relacionados con la autonomía que el estudiante adquiere y desarrolla. Así, el 72,3\% afirma (valorando el ítem con 4 o 5) que esta metodología favorece el autoaprendizaje del alumnado y un $70,8 \%$ (4 o 5) señalan que la autorregulación es muy importante en estos modelos, con una manera de trabajar más autónoma y en un entorno más activo.

Al valorar la importancia de las dos modalidades que conforman el aprendizaje híbrido (presencial y virtual) parece que no se decantan claramente por la eficacia de ninguna de las dos sino que parece que lo ideal es una mezcla más o menos homogénea de ambas modalidades.

\section{Aprendizaje presencial Vs virtual}

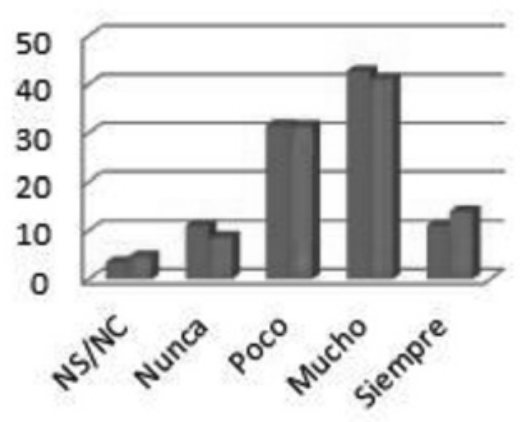

En mi aprendizaje
combinado
(presencial/virtual)
ha sido más eficaz
lo presencial
En mi aprendizaje
combinado
(presencial/virtual)
ha sido más eficaz
lo virtual

Figura 8. Comparación entre la eficacia del aprendizaje virtual y presencial en blended learning

En la pregunta sobre si elegirían una modalidad exclusivamente presencial la mayoría se decanta por no escogerla eligiendo un $65.9 \%$ los valores «Nunca» y «Poco». Se podría decir que una modalidad combinada les resulta más atractiva que una que es solamente presencial. 


\section{Elijo modalidad exclusivamente presencial}
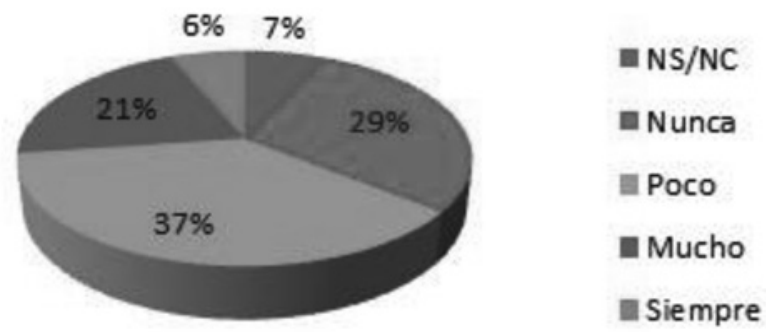

Figura 9. Preferencias en la modalidad presencial/virtual

En general, la valoración que hacen de esta modalidad una vez terminada la experiencia es bastante positiva, escogiendo un 51,2\% el valor de Buena y un 22,7 la de muy buena. Tan sólo un 23,2\% la percibe como Mala o Regular.

La información cualitativa recogida en foros y a través de la parte cualitativa del cuestionario CVMA complementa estos resultados. A continuación se muestran estos resultados para cuyo análisis se realizó un proceso de reducción y extracción de categorías basado en la literatura completado con las categorías derivadas de la lectura detallada de la información textual recogida. Categorías y frecuencias sobre la modalidad de aprendizaje en foro y cuestionario aparecen en la Tabla 2.

Tabla 2

Tabla de frecuencias de categorías en foro y cuestionario sobre la modalidad de enseñanzaaprendizaje

MODELO DE ENSEÑANZAAPRENDIZAJE

\begin{tabular}{|c|c|c|c|}
\hline $\begin{array}{l}\text { Destacan las herramientas de comu- } \\
\text { nicación, chat y foro }\end{array}$ & MODGEN_DES_COM & 15 & 26 \\
\hline $\begin{array}{l}\text { Permite entregar los trabajos de } \\
\text { manera ordenada (Secuenciación en } \\
\text { la entrega de trabajos) }\end{array}$ & MODGEN_SEC_TRA & 2 & 22 \\
\hline Flexibilidad espacio temporal & MODGEN_FLEXIB & 2 & 15 \\
\hline $\begin{array}{l}\text { Aprendizaje de competencias tecno- } \\
\text { lógicas gracias a la nueva plataforma }\end{array}$ & MODGEN_APREN_TEC & - & 14 \\
\hline $\begin{array}{l}\text { Permite el trabajo autónomo e inde- } \\
\text { pendiente del estudiante }\end{array}$ & MODGEN_TRAB_AUT & 9 & - \\
\hline
\end{tabular}


En primer lugar, los estudiantes destacan las posibilidades que ofrecen las herramientas de comunicación (foro y chat). Vuelve a aparecer en este caso la mejora de la comunicación entre estudiantes y profesores como una de las principales ventajas para facilitar sus tareas y ayudarles en sus procesos de aprendizaje.

Otro aspecto positivo destacado por los estudiantes es la flexibilidad espacio-temporal de esta metodología, por ejemplo, para el acceso a recursos y la entrega de actividades de manera secuenciada y ordenada, o para la utilización como medio ideal para el aprendizaje de competencias tecnológicas.

Otra opinión reiterada es que este método favorece el trabajo autónomo e independiente del estudiante. Consideran que estas metodologías hacen posible que el estudiante pueda trabajar más por cuenta propia pero sin perder el contacto con el grupo ni con el profesor que ejerce de guía en el proceso de aprendizaje como ya comentábamos anteriormente.

En cuanto a las características negativas, algunos afirman que con esta modalidad se pierde quizá el trato más personal que da la presencialidad sobre todo en las experiencias que tienen más carga virtual que presencial. También hay algunos que opinan que, al contrario de lo que podría parecer, con estas metodologías el trabajo realizado y el esfuerzo empleado es mayor que en las asignaturas únicamente presenciales, como ya advertían en los cuestionarios. Cuando tienen que señalar lo peor de la experiencia se centran principalmente en dos aspectos: en primer lugar tienen un cierto «Estrés por la rigidez en la planificación del modelo, por no poder entregar las actividades a tiempo o no poder participar en los chats...» y además perciben que este tipo de metodologías «Requiere mayor trabajo». Sin embargo, siguen teniendo la percepción de que existe diferencia entre su carga de trabajo y la del docente, como ya se señaló en los datos cuantitativos.

En resumen, podemos decir que los estudiantes destacan positivamente en esta metodología las características más significativas para la consolidación del EEES. Entre estas destacan las facilidades que aportan para la comunicación, el fomento del trabajo autónomo y colaborativo y la flexibilidad y disponibilidad en cualquier momento (Álvarez, Guash y Espasa, 2009). 


\section{Participación de los estudiantes: análisis de estadísticas de acceso}

Otro indicador para analizar la metodología empleada es el grado de participación e intervención que los estudiantes muestran en las experiencias semipresenciales. A continuación se contrastan algunos índices de participación del alumnado en la plataforma con las calificaciones obtenidas en sus trabajos para intentar establecer relaciones.

Para realizar este análisis se han utilizado datos procedentes de dos grupos de estudiantes de asignaturas en las que se utilizaron todos los módulos de interacción y participación de la plataforma (incluidos comunidades virtuales y autoevaluaciones). Analizamos las estadísticas de 122 estudiantes de los cuales 67 pertenecen al primer grupo y 55 al segundo.

En la línea de otros trabajos de análisis de la relación entre participación y rendimiento de los estudiantes (Cuadrado, Ruiz Molina y Coca, 2009) se escogieron para la presente investigación los indicadores de participación que podrían aportar el grado de implicación que los estudiantes tienen en esta modalidad de enseñanza:

- Número de conexiones totales a la plataforma.

- Número de accesos a la sección de Comunidades (trabajo en equipo.

- Número de intervenciones en el foro.

Como valoración del resultado final se seleccionan tres valores característicos de la evaluación que tendría el estudiante al finalizar la asignatura:

- Nota media obtenida en tres actividades de clase (obligatorias).

- Nota media obtenida en el apartado de Comunidades (trabajo en equipo).

- Nota obtenida en un ejercicio de autoevaluación antes del examen oficial.

Una vez definidas estas variables para determinar la correlación entre participación e implicación de los estudiantes y su rendimiento académico se utilizó el coeficiente de concordancia por rangos de Spearman. En la Tabla 3 se muestran los resultados obtenidos al realizar esta prueba con el programa estadístico SPSS. 
Tabla 3

Correlaciones entre los índices de participación y los resultados académicos de los estudiantes

\begin{tabular}{lccc}
\hline \multicolumn{4}{c}{ Correlaciones } \\
\hline & Media_trabajos & Media_comunidades & Autoevaluacion \\
\hline $\begin{array}{l}\text { Numero_accesos } \\
\text { Numero_accesos }\end{array}$ & $.413(* *)$ & $.223(*)$ & $.386(* *)$ \\
$\begin{array}{l}\text { Intervenunidades } \\
\text { foro }\end{array}$ & $.432(* *)$ & $.287(* *)$ & $.366(* *)$ \\
\hline
\end{tabular}

** La correlación es significativa al nivel .01 (bilateral).

* La correlación es significativa al nivel .05 (bilateral).

Se obtienen relaciones positivas y significativas entre las variables cuantificadoras del grado de implicación de los estudiantes en la experiencia y las de sus resultados académicos para todas las combinaciones excepto para el binomio Autoevaluación-Intervenciones_foro. De manera que se puede concluir que cuanto mayor es el nivel de participación y acceso a la plataforma, mejores son los resultados obtenidos por los estudiantes. Resultados similares se han obtenido en los estudios de Cuadrado, Ruiz Molina y Coca (2009), Cabero y Llorente (2009) o De la Fuente y otros (2007).

También se ha querido observar la evolución en el rendimiento académico de los estudiantes para poder determinar la incidencia de este modelo en sus resultados finales. Por disponibilidad de datos previos al empleo sistemático de la metodología híbrida del presente estudio en la asignatura «Tecnología Educativa»se ha elaborado la Figura 10 en la que se observa cómo la nota media de los estudiantes de esta asignatura aumenta con la introducción del modelo semipresencial en el curso académico 2007-2008 y 2008-2009. 


\section{Evolución de la nota media en la asignatura "Tecnología Educativa" \\ - NOTA MEDIA}

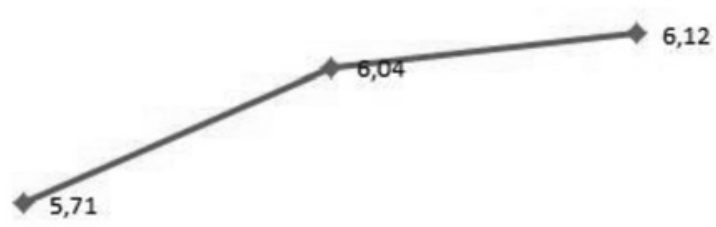

2006-2007

$2007-2008$

2008-2009

Figura 10. Evolución en la nota media de los estudiantes de la asignatura «Tecnología Educativa» de la licenciatura de Pedagogía

\section{CONCLUSIONES}

El proceso de convergencia que lleva a las universidades al EEES demanda nuevas estrategias formativas que posibiliten esta transición. Las metodologías basadas en el aprendizaje y centradas en el estudiante, el trabajo del estudiante que pasa a ser más autónomo o la flexibilidad de una formación a lo largo de toda la vida, son sólo algunos de esos elementos de cambio. En este proceso, parece que las metodologías didácticas basadas en las TIC tienen las propiedades necesarias para convertirse en elementos importantes.

De los datos obtenidos en nuestra experimentación con metodologías blended learning se pueden extraer algunas conclusiones en torno a varias líneas fundamentales relacionadas con la puesta en práctica de las metodologías combinadas. En primer lugar, en relación con su trabajo en esta modalidad, los estudiantes piensan que la metodología empleada favorece el autoaprendizaje y el trabajo autónomo del alumnado y le dan mucha importancia a la autorregulación, consideran seriamente que con esta modalidad y a través de la plataforma es más sencillo que se participe en las distintas actividades propuestas fomentando el aprendizaje activo y sirviendo para desarrollar actividades prácticas. Se sienten protagonistas y responsables de su propio aprendizaje y creen estar preparados para afrontarlo aunque quizá con algo de formación que podrán alcanzar de manera autónoma. Del mismo modo, señalan como una característica destacada de la modalidad semipresencial la flexibilidad horaria y espacial que proporciona y la posibilidad de acceder a los recursos disponibles en cualquier momento y 
desde cualquier ubicación. Destacan también las posibilidades que ofrecen las herramientas de comunicación.

En cuanto al papel del docente perciben su actuación como mediador y guía en el proceso de aprendizaje, para lo cual la comunicación y la coordinación entre todas las partes es determinante. Creen, por otra parte, que el docente desempeña la misma función en docencia presencial y no presencial, percepción que podría derivar en una simple transferencia del modelo presencial al modelo no presencial, copiando los mismos patrones sin adaptar verdaderamente la metodología a los nuevos escenarios, lo cual no sería deseable para el cambio. La importancia que para el estudiante tiene identificarse con la forma de trabajo del profesor es el criterio orientador de lo que García-Valcárcel y Tejedor (2011) denominan «escenario de aprendizaje».

Entre los aspectos negativos destaca que los estudiantes consideran que el trabajo realizado en una asignatura semipresencial es mayor que el realizado en otras totalmente presenciales. Esta percepción puede ser debida a la transición en el cambio de paradigma que puede desembocar en esos desajustes entre el trabajo potencial y el trabajo real. Sin embargo, parece por los resultados obtenidos que la participación de los estudiantes en una asignatura semipresencial está relacionada con los resultados que obtienen en esa asignatura.

También aprecian una falta de atención individualizada del profesorado, característica sin duda muy importante de la enseñanza online, pero muy lejos de la realidad del sistema universitario actual teniendo en cuenta la masificación de los grupos y los reajustes que la crisis provoca, siendo muy difíciles las tareas de seguimiento.

En general, cuando se trata de observar el efecto de la tecnología, los meta-análisis publicados proporcionan información valiosa pero ni uno solo es capaz de responder a la cuestión general del impacto global del uso de la tecnología en el rendimiento de los estudiantes. Aunque el promedio de los estudiantes en un aula con tecnología podría ser hasta 12 puntos porcentuales más alto que el de los que se encuentran en un ambiente tradicional que no utiliza la tecnología, Tamin et al. (2011) advierten que estos efectos promedio deben interpretarse con precaución debido a la gran variabilidad que los rodea, pudiendo ser debidos a otros factores no identificados en dichos metanálisis. Probablemente sean más debido al método que al medio. Similar es el resultado de la investigación de Emerson y McKay (2011) quienes al comparar encuentran que la lección sobre el papel llega a resultar un 24\% mejor que la misma lección online. Existen dificultades para establecer conclusiones fiables en estudios que comparan las formas 
tradicionales de aprendizaje (por ejemplo, en papel o en el aula) versus aprendizaje en línea.

Si los resultados de la investigación no son consistentes, habría que tenerlo en cuenta de cara a las decisiones de las instituciones de educación superior acerca del aprendizaje online y del blended learning. En cualquier caso, la investigación sobre el blended learning sigue creciendo (Drysdale et al., 2013) y se diversifica en ciencias naturales (Andrade, 2007), económicas (López, Pérez y Rodríguez, 2011), geografía (Mitchell y Forer, 2010) e ingeniería (Francis y Shannon, 2013).

La proyección que una didáctica universitaria general puede realizar debe ir dirigida hacia el aprovechamiento de modelos como estos para fomentar el aprendizaje activo a través de actividades prácticas y comunidades de aprendizaje virtual fomentando la comunicación a través de las posibilidades de internet; la explotación de los mecanismos de autorregulación que puede aportar la web tales como ejercicios de autoevaluación o rúbricas. Es necesario que el docente ajuste su metodología para no provocar desajustes en el tiempo de trabajo del estudiante ni del docente y que potencie su papel de guía y dinamizador en estos entornos. En definitiva, sería necesario mejorar la atención individualizada aunque es difícil con grupos de tamaño grande.

Las potencialidades mencionadas por los estudiantes (fomento del trabajo autónomo, aprendizaje activo, flexibilidad...) coinciden con los principios metodológicos que se promueven en la reforma de la Educación Superior. Con todo ello, se puede concluir que la metodología usada basada en el blended learning, conforme al modelo de análisis seguido, resulta beneficiosa para el proceso de convergencia hacia el EEES. 


\section{REFERENCIAS BIBLIOGRÁFICAS}

Álvarez, I., Guash, T., y Espasa, A. (2009). University teacher roles and competencies in online learning environments: a theoretical analysis of teaching and learning practices. European Journal of Teacher Education, 32 (3), 321-336. doi: 10.1080/02619760802624104

Andrade, A. (2007). Aprendizaje combinado como propuesta en la convergencia europea para la enseñanza de las ciencias naturales. eLearning Papers, 3, 1-12.

Cabero, J. y Llorente, M.C. (2009). Actitudes, satisfacción, rendimiento académico y comunicación online en procesos de formación universitaria en blended learning. Revista Electrónica Teoría de la Educación. Educación y Cultura en la Sociedad de la Información, 10(1), 172-189.

Campbell, D.T. y Fiske, D. (1959). Convergent and discriminant validation by the multitrait-multimethod matrix. Psychological Bulletin, 56, 81-105. doi: 10.1037/h0046016

Castaño-Muñoz, J., Duart, J.M., y Sancho-Vinuesa, T. (2014). The Internet in face-to-face higher education: Can interactive learning improve academic achievement? British Journal of Educational Technology, 45(1), 149-159. doi: 10.1111/bjet.12007

Cuadrado, M.F., Ruiz Molina, M.E., y Coca, M. (2009). Participación y rendimiento del estudiante universitario en un proyecto docente interdisciplinar, bilingüe y virtual. Revista de Educación, 348, 505-518.

De la Fuente, J., Cano, F., Justicia, F., Pichardo, M.C., García-Berbén, A.B., Martínez-Vicente, J.M. y Sanders, P. (2007). Efectos de usar herramientas electrónicas en la mejora de la regulación del proceso enseñanza-aprendi- zaje. Electronic journal of research in educational psychology, 5(3), 757-782.

Delialioglu, O. y Yildirim, Z. (2008). Design and development of a technology enhanced hybrid instruction based on MOLTA model: Its effectiveness in comparison to traditional instruction. Computers \& Education, 51, 474-483. doi: 10.1016/j.compedu.2007.06.006

Diario Oficial de la UE (2011). Conclusiones del Consejo sobre la modernización de la enseñanza superior (2011/C 372/09). Recuperado de http://eur-lex. europa.eu/LexUriServ/LexUriServ.do? uri=OJ:C:2011:372:0036:0041:ES:PDF

Drysdale, J.S., Graham, C.R., Spring, K.J., y Halverson, L.R. (2013). An analysis of research trends in dissertations and theses studying blended learning. Internet and Higher Education, 17, 90-100. doi: 10.1016/j.iheduc. 2012.11 .003

Emerson, L. y MacKay, B. (2011). A comparison between paper-based and online learning in higher education. British Journal of Educational Technology, 42(5), 727-735. doi: 10.1111/j.1467-8535.2010.01081.x

Fabian, P., Veen, W., y Mühren, A. (2008). Experience gained from the EU Socrates/Minerva project: "Finding a balance in blended learning with extra large student groups". eLearn 2008, ZbornikPríspevkov, 13-14 May.

Francis, R. y Shannon, S.J. (2013). Engaging with blended learning to improve students' learning outcomes. European Journal of Engineering Education, 38(4), 1-11.

Gallego, M.J. y Gámiz, V. (2014). Personal Learning Environments (PLE) in the Academic Achievement of Univer- 
sity Students. Australian Educational Computing, 29(2).

Gallego, M.J. y Gámiz, V. (2007). Un camino hacia la innovación basada en un entorno de aprendizaje virtual aplicado a la inmersión práctica en los estudios universitarios de educación. RELATEC - Revista Latinoamericana de Tecnología Educativa, 6(1), 13-31.

García-Valcárcel, A. y Tejedor, F.J. (2011). Variables TIC vinculadas a la generación de nuevos escenarios de aprendizaje en la enseñanza universitaria. Aportes de las curvas ROC para el análisis de diferencias. Educación XX1, 14 (2), 43-78.

Ginns, P. y Ellis, R. A. (2009). Evaluating the quality of e-learning at the degree level in the student experience of blended learning. British Journal of Educational Technology, 40 (4), 652-663. doi: 10.1111/j.1467-8535.2008.00861.x

Graham, C.R. (2006). Blended Learning Systems: Definitions, current trends and Future Directions. En C. Bonk y C.R. Graham (Eds.) The Handbook of Blended Learning. Global Perspectives, Local Designs (pp.3-21). San Francisco: Pfeiffer.

Lloret, T. y Mir, A. (2007) ¿Qué ha ocurrido en el primer año de implementación del EEES en algunas titulaciones?: Un primer balance en la UPF en términos de rendimiento académico, satisfacción y proceso de enseñanza aprendizaje. Red U. Revista de Docencia Universitaria, 5(1), 1-17.

López-Pérez, M.V., Pérez-López, M.C. y Rodríguez-Ariza, L. (2011). Blended learning in higher education: Students' perceptions and their relation to outcomes. Computers \& Education, 56, 818826. doi: 10.1016/j.compedu.2010.10.023

Mitchell, P. y Forer, P. (2010). Blended learning: the perceptions of first-year geography students. Journal of Geography in Higher Education, 34 (1), 77-89. doi: 10.1080/03098260902982484

Montil, M., Barcelo, O., Bielsa, R., y Oliván, J. (2007). Análisis del trabajo no presencial utilizando un enfoque ETCS. Revista complutense de educación, 18(1), 179-196.

Rovai, A.P., Ponton, M.K., Derrick, M.G., y Davis, J.M. (2006). Student evaluation of teaching in the virtual and traditional classrooms: A comparative analysis. The Internet and Higher Education, 9 (1), 23-35. doi: 10.1016/j. iheduc.2005.11.002

Ryberg, T., Niemczik, C., y Brenstein, E. (2009). Methopedia - Pedagogical Design Community for European Educators. En D. Remenyi (Ed.), The Proceedings of the 8th European Conference on e-Learning (pp. 503-511). Recuperado de http://www.academia. edu/554490/Methopedia-Pedagogical_ Design_Community_for_European_ Educators

Tamim, R.M., Bernard, R.M., Borokhovski, E., Abrami, P.C., y Schmid, R.F. (2011). What Forty Years of Research Says About the Impact of Technology on Learning: A Second-Order MetaAnalysis and Validation Study. Review of Educational Research, 81(1), 4-28. doi: 10.3102/0034654310393361

Uceda, J. y Píriz, S. (Coord.) (2012). UNIVERSITIC 2012: Descripción, gestión y gobierno de las TI en el SUE. Madrid: Conferencia de Rectores de las Universidades Españolas (CRUE). Recuperado de http://www.crue.org/export/ sites/Crue/Publicaciones/Documentos/ Universitic/UNIVERSITIC2012baja.pdf

Webb, E.J., Campbell, D.T., Schwartz, R.D. y Sechrest, L. (1966). Unobstrusive measures: nonreactive research in the social sciences. Chicago: Rand McNally. 


\section{PERFIL ACADÉMICO Y PROFESIONAL DE LOS AUTORES}

Vanesa Gámiz-Sánchez, Ingeniera Informática por la Universidad de Granada en 2003 y doctora en Ciencias de la Educación por la misma universidad con el trabajo «Entornos Virtuales para la formación práctica de estudiantes de educación». Actualmente contratada como profesora en la Facultad de Ciencias de la Educación. Participa en varias investigaciones sobre el uso de las TIC en procesos de enseñanza-aprendizaje (e-learning, entornos personales de aprendizaje, recursos educativos abiertos, e-portafolios, seguridad en Internet).

María-Jesús Gallego-Arrufat, Profesora Titular de la Universidad de Granada con acreditación como Catedrática de Universidad (ANECA). Doctora en Filosofía y Ciencias de la Educación. Docente en la especialidad de Tecnología Educativa. Sus principales líneas de investigación son las TIC en educación, la formación del profesorado y el prácticum. Estudia la didáctica universitaria y la comunicación en entornos virtuales de aprendizaje, el empleo de recursos TIC en educación superior (comunicación mediada por ordenador, e-rúbricas, entornos personales de aprendizaje, mooc) y el liderazgo en entornos virtuales de formación.

Dirección de las Autoras: Departamento de Didáctica y Organización Escolar

Facultad de Ciencias de la Educación

Campus de Cartuja, s/n

Universidad de Granada

18071 Granada, (Spain)

Email: vanesa@ugr.es

mgallego@ugr.es

Fecha Recepción del Artículo: 8. Enero. 2013

Fecha modificación Artículo: 25. Marzo. 2013

Fecha Aceptación del Artículo: 28. Mayo. 2013

Fecha Revisión para Publicación: 05. Julio. 2015 
